\title{
Near-Capacity Three-Stage Turbo Detection of Irregular Convolutional Coded Joint Sphere-Packing Modulation and Space-Time Coding
}

\author{
O. Alamri, J. Wang, S. X. Ng, L.-L. Yang, and L. Hanzo
}

\begin{abstract}
Conventional two-stage turbo-detected schemes typically suffer from a Bit Error Rate (BER) floor, preventing them from achieving infinitesimally low BER values, especially, when the inner coding stage is of non-recursive nature. We circumvent this deficiency by proposing a three-stage turbo-detected Sphere Packing (SP) aided Space-Time Block Coding (STBC) STBC-SP scheme, where a rate-1 recursive inner precoder is employed to avoid having a BER floor. The convergence behaviour of this serially concatenated scheme is investigated with the aid of 3D Extrinsic Information Transfer (EXIT) Charts. Furthermore, the capacity of the STBC-SP scheme is determined and an algorithm is proposed for calculating a tighter upper bound on the maximum achievable bandwidth efficiency, based on the EXIT charts of the STBC-SP demapper. The proposed threestage turbo-detected scheme operates within about $1.0 \mathrm{~dB}$ of the capacity and within $0.5 \mathrm{~dB}$ of the maximum achievable bandwidth efficiency limit.
\end{abstract}

Index Terms-

\section{INTRODUCTION}

$\mathbf{T}$ HE adverse effects of channel fading may be significantly reduced by employing orthogonal transmit diversity invoking multiple antennas $[1,2]$. The concept of combining orthogonal transmit diversity designs with the principle of sphere packing was introduced by Su et al. in 2003 [3] in order to maximise the achievable coding advantage ${ }^{1}$, where it was demonstrated that the proposed Sphere Packing (SP) aided Space-Time Block Coded (STBC) system, referred to here as STBC-SP, was capable of outperforming the conventional orthogonal design based STBC schemes of $[4,5]$. The ultimate rationale of this paper is to use a novel three-dimensional Extrinsic Information Transfer (EXIT)-chart-based technique to jointly design the two time-slots' STBC signal by nearoptimally combining them into an iteratively detected SP symbol.

The turbo principle of [6] was extended to multiple parallel concatenated codes [7], to serially concatenated codes [8] as

Paper approved by A. H. Banihashemi, the Editor for Coding and Communication Theory of the IEEE Communications Society. Manuscript received June 23, 2006; revised January 14, 2008 and August 23, 2008.

The financial support of the EPSRC, UK and that of the European Community under Seventh Framework Programme grant agreement ICT OPTIMIX nINFSO-ICT-214625, as well as that of the Ministry of Higher Education of Saudi Arabia, is gratefully acknowledged.

The authors are with the School of ECS, University of Southampton, United Kingdom (e-mail: lh@ecs.soton.ac.uk).

Digital Object Identifier 10.1109/TCOMM.2009.05.060377

${ }^{1}$ The diversity product or coding advantage was defined as the estimated gain over an uncoded system having the same diversity order as the coded system [3] well as to multiple serially concatenated codes [9]. The appeal of concatenated coding is that low-complexity iterative detection replaces the potentially more complex optimum decoder, such as that of [10]. In [11], the employment of the turbo principle was considered for iterative soft demapping in the context of bit-interleaved coded modulation (BICM), where a soft demapper was used between the multilevel demodulator and the channel decoder. In [12], a turbo coding scheme was proposed for the multiple-input multiple-output (MIMO) Rayleigh fading channel, where a block code was employed as an outer channel code, while an orthogonal STBC scheme was considered as the inner code. The iterative soft demapping principle of [11] was extended to STBC-SP schemes in $[2,13-15]$, where it was demonstrated that turbo-detected STBC-SP schemes provide useful performance improvements over conventionally-modulated orthogonal design based STBC schemes. It was shown in [16] that a recursive inner code is needed in order to maximise the interleaver gain and to avoid the formation of a bit-error rate (BER) floor, when employing iterative decoding. This principle has been adopted by several authors designing serially concatenated schemes, where rate1 inner codes were employed for designing low complexity turbo codes suitable for bandwidth and power limited systems having stringent BER requirements [17-21].

Recently, studying the convergence behaviour of iterative decoding has attracted considerable attention [22-26]. In [22], ten Brink proposed the employment of the so-called EXIT characteristics between a concatenated decoder's output and input for describing the flow of extrinsic information through the soft-in/soft-out constituent decoders. The computation of EXIT charts was further simplified in [23] to a time average, for scenarii when the PDFs of the communicated information at the input and output of the constituent decoders are both symmetric and ergodic. The concept of EXIT chart analysis has been extended to three-stage concatenated systems in [2426].

In this paper, we propose a capacity-approaching three-stage turbo-detected STBC-SP scheme, where iterative decoding is carried out between three constituent decoders, namely an STBC-SP demapper, an inner rate-1 recursive A Posteriori Probability (APP)-based decoder and an outer APP-based decoder. We first derive the capacity limit for STBC-SP schemes. Then, an upper bound on the maximum achievable rate is calculated, based on the EXIT charts of the STBCSP demapper. At a spectral efficiency of $\eta=1 \mathrm{bits} / \mathrm{s} / \mathrm{Hz}$, the 

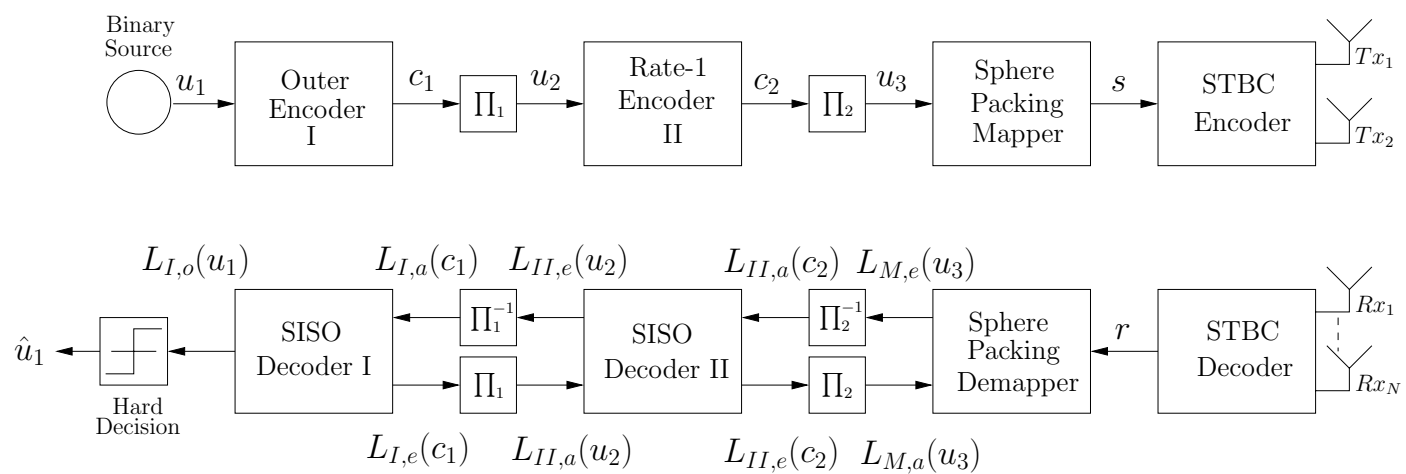

Fig. 1. Three-stage serially concatenated system.

upper bound of the maximum achievable rate is within $0.5 \mathrm{~dB}$ of the capacity, and our proposed three-stage scheme operates within $1.0 \mathrm{~dB}$ of the capacity. The rationale of the proposed architecture is explicit: (1) SP modulation maximises the coding advantage of the transmission scheme by jointly designing and detecting the SP symbols hosting the two time-slots' STBC symbols; (2) the inner rate-1 recursive decoder maximises the interleaver gain and hence avoids having a BER floor; and (3) the outer irregular convolutional codes (IRCCs) [23,27] minimise the area of the EXIT chart's convergence tunnel and hence facilitate near-capacity operation [28].

This paper is organised as follows. In Section II, a brief description of our three-stage system is presented. Section III provides our 3D EXIT chart analysis along with its simplified 2D projections. The capacity of STBC-SP schemes is derived in Section IV, where an upper bound on the maximum achievable rate is also calculated based on the EXIT chart analysis. Our simulation results and discussions are provided in Section V. Finally, we conclude in Section VI.

\section{SySTEM OVERVIEW}

\section{A. Encoder}

The schematic of the entire system is shown in Figure 1, where the transmitted source bits $\mathbf{u}_{1}$ are encoded by the outer channel Encoder I having a rate of $R_{I}$. The outer channel encoded bits $\mathbf{c}_{1}$ are then interleaved by the first random bit interleaver, where the randomly permuted bits $\mathbf{u}_{2}$ are fed through the rate- 1 Encoder II. The concatenated coded bits $\mathbf{c}_{2}$ at the output of the rate- 1 encoder are interleaved by the second random bit interleaver, producing the permuted bits $\mathbf{u}_{3}$. After channel interleaving, the sphere packing mapper first maps blocks of $B$ channel-coded bits $\mathbf{b}=b_{0, \ldots, B-1} \in\{0,1\}$ to the $L=2^{B}$ number of legitimate four-dimensional sphere packing modulated symbols $\mathbf{s}^{l} \in S$, where $S=\left\{\mathbf{s}^{l}=\left[a_{l, 1}\right.\right.$ $\left.\left.a_{l, 2} \quad a_{l, 3} a_{l, 4}\right] \in \mathbb{R}^{4}: 0 \leq l \leq L-1\right\}$ constitutes a set of $L$ legitimate constellation points from the lattice $D_{4}$ [29] having a total energy of $E \triangleq \sum_{l=0}^{L-1}\left(\left|a_{l, 1}\right|^{2}+\left|a_{l, 2}\right|^{2}+\left|a_{l, 3}\right|^{2}+\left|a_{l, 4}\right|^{2}\right)$. The STBC encoder then maps each sphere packing modulated symbol $\mathbf{s}^{l}$ to a space-time signal $\mathbf{C}_{l}$ as $[3,13]$ :

$$
\mathbf{C}_{l}=\sqrt{\frac{2 L}{E}} \mathbf{G}_{2}\left(x_{l, 1}, x_{l, 2}\right), \quad 0 \leq l \leq L-1,
$$

where $x_{l, 1}$ and $x_{l, 2}$ are complex-valued symbols constructed from the 4-dimensional real-valued coordinates of the SP symbol $\mathbf{s}^{l}$ in order to maximise the coding advantage of the space-time signal $\mathbf{C}_{l}$ [3], since the lattice $D_{4}$ has the best minimum Euclidean distance in the four-dimensional realvalued Euclidean space $\mathbb{R}^{4}$ [29]. Specifically, $x_{l, 1}$ and $x_{l, 2}$ may be written as

$$
\begin{aligned}
\left\{x_{l, 1}, x_{l, 2}\right\} & =T_{s p}\left(a_{l, 1}, a_{l, 2}, a_{l, 3}, a_{l, 4}\right) \\
& =\left\{a_{l, 1}+j a_{l, 2}, a_{l, 3}+j a_{l, 4}\right\} .
\end{aligned}
$$

Furthermore, $\mathbf{G}_{2}\left(x_{l, 1}, x_{l, 2}\right)$ is the space-time transmission matrix given by [4]

$$
\mathbf{G}_{2}\left(x_{1}, x_{2}\right)=\left[\begin{array}{cc}
x_{1} & x_{2} \\
-x_{2}^{*} & x_{1}^{*}
\end{array}\right],
$$

where the rows and columns of Eq. (3) represent the temporal and spatial dimensions, corresponding to two consecutive time slots and two transmit antennas, respectively.

\section{B. Channel Model}

In this treatise, we considered a time-correlated narrowband Rayleigh fading channel, based on Jakes' fading model [30] associated with a normalised Doppler frequency of $f_{D}=$ $f_{d} T_{s}=0.1$, where $f_{d}$ is the Doppler frequency and $T_{s}$ is the symbol period. However, the complex-valued fading envelope is assumed to be constant only across the transmission period of a space-time coded symbol spanning $T=2$ time slots and varies from one symbol to another according to the aforementioned Rayleigh fading model. The complex Additive White Gaussian Noise (AWGN) of $n=n_{I}+j n_{Q}$ is also added to the received signal, where $n_{I}$ and $n_{Q}$ are two independent zero-mean Gaussian random variables having a variance of $\sigma_{n}^{2}=\sigma_{n_{I}}^{2}=\sigma_{n_{Q}}^{2}=N_{0} / 2$ per dimension, where $N_{0} / 2$ represents the double-sided noise power spectral density expressed in $W / H z$.

\section{Decoder}

As shown in Figure 1, the received complex-valued symbols are first decoded by the STBC decoder in order to produce the received SP soft-symbols $r$, where each SP symbol represents a block of $B$ coded bits [13]. Then, iterative demapping/decoding is carried out between the SP demapper, APP-based soft-in/soft-out (SISO) module II and APP-based SISO module I, where extrinsic information is exchanged between the three constituent demapper/decoder 


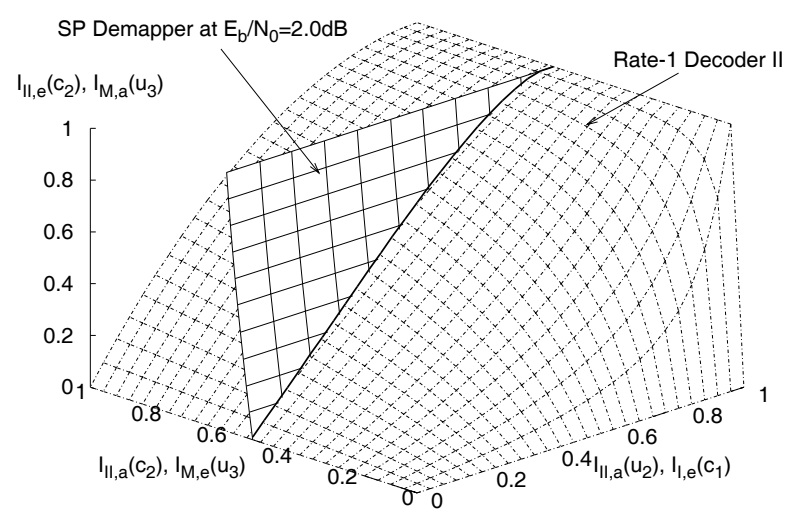

Fig. 2. 3D EXIT chart of Decoder II and the SP demapper at $E_{b} / N_{0}=$ $2.0 \mathrm{~dB}$.

modules. More specifically, $L_{\cdot, a}(\cdot)$ in Figure 1 represents the a priori information, expressed in terms of the log-likelihood ratios (LLRs) of the corresponding bits, whereas $L_{\cdot, o}(\cdot)$ and $L_{., e}(\cdot)$ represent the a posteriori and extrinsic LLRs of the corresponding bits, respectively, where the subscript $(\cdot)$ is used to distinguish the different constituent decoders, i.e. Decoder I, Decoder II and the SP demapper. The iterative process is performed for a number of consecutive iterations. During the last iteration, only the a posteriori LLR values $L_{I, o}\left(\mathbf{u}_{\mathbf{1}}\right)$ of the original uncoded systematic information bits $\mathbf{u}_{1}$ are required, which are passed to a hard decision decoder in order to determine the estimated transmitted source bits $\hat{\mathbf{u}}_{1}$ as shown in Figure 1.

\section{EXIT CHART ANALYSIS}

\section{A. Preliminaries}

The main objective of employing EXIT charts [22], is to predict the convergence behaviour of the iterative decoder by examining the evolution of the input/output mutual information exchange between the inner and outer decoders in consecutive iterations. The application of EXIT charts is based on the two assumptions that upon assuming large interleaver lengths, (1) the a priori LLR values are fairly uncorrelated; (2) the a priori LLR values exhibit a Gaussian PDF. In this section, the approach presented in [26] is adopted in order to provide the EXIT chart analysis of the proposed three-stage system of Figure 1.

Let $I_{\cdot, a}(x), 0 \leq I_{\cdot, a}(x) \leq 1$, denote the mutual information (MI) [31] between the a priori LLRs $L_{\cdot, a}(x)$ as well as the corresponding bits $x$ and let $I_{\cdot, e}(x), 0 \leq I_{\cdot, e}(x) \leq 1$, denote the MI between the extrinsic LLRs $L_{,, e}(x)$ and the corresponding bits $x$.

\section{B. 3D EXIT Charts}

As seen from Figure 1, the input of Decoder II is constituted by the a priori input $L_{I I, a}\left(c_{2}\right)$ and the a priori input $L_{I I, a}\left(u_{2}\right)$ provided after bit-deinterleaving by the SP demapper and Decoder I, respectively. Therefore, the EXIT characteristic of Decoder II can be described by the following

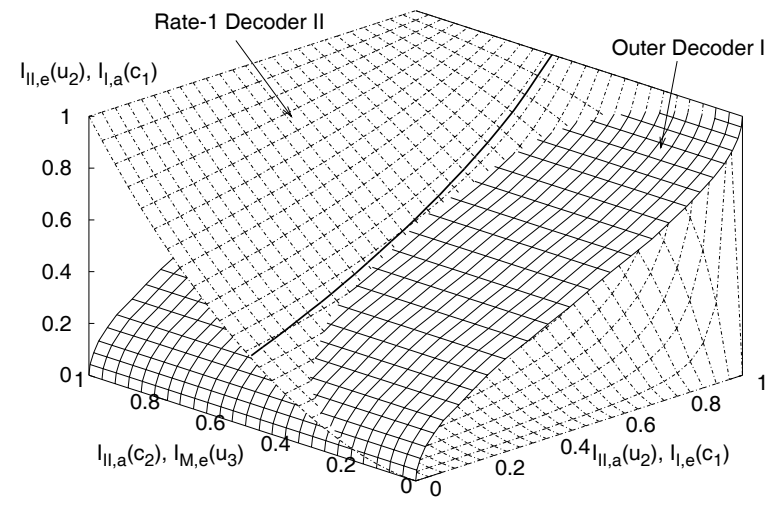

Fig. 3. 3D EXIT chart of Decoder II and Decoder I with projection from Figure 2.

two EXIT functions [22,26]:

$$
\begin{aligned}
& I_{I I, e}\left(c_{2}\right)=T_{I I, c_{2}}\left(I_{I I, a}\left(u_{2}\right), I_{I I, a}\left(c_{2}\right)\right), \\
& I_{I I, e}\left(u_{2}\right)=T_{I I, u_{2}}\left(I_{I I, a}\left(u_{2}\right), I_{I I, a}\left(c_{2}\right)\right),
\end{aligned}
$$

which are illustrated by the 3D surfaces drawn in dotted lines in Figures 2 and 3, respectively. On the other hand, the EXIT characteristic of the SP demapper as well as that of Decoder I are each dependent on a single a priori input, namely on $L_{M, a}\left(u_{3}\right)$ and $L_{I, a}\left(c_{1}\right)$, respectively, both of which are provided by the rate-1 Decoder II after appropriately ordering the bits, as seen in Figure 1. The EXIT characteristic of the SP demapper is also dependent on the $E_{b} / N_{0}$ value. Consequently, the corresponding EXIT functions for the SP demapper and Decoder I, respectively, may be written as

$$
\begin{aligned}
I_{M, e}\left(u_{3}\right) & =T_{M, u_{3}}\left(I_{M, a}\left(u_{3}\right), E_{b} / N_{0}\right), \\
I_{I, e}\left(c_{1}\right) & =T_{I, c_{1}}\left(I_{I, a}\left(c_{1}\right)\right),
\end{aligned}
$$

which are illustrated by the 3D surfaces drawn in solid lines in Figures 2 and 3, respectively.

Eqs. (4) to (7) may be represented with the aid of two 3D EXIT charts. More specifically, the 3D EXIT chart of Figure 2 can be used to plot Eq. (4) and Eq. (6), which describe the EXIT relation between the SP demapper and Decoder II. Similarly, the 3D EXIT chart of Figure 3 can be used to describe the EXIT relation between Decoder II and Decoder I by plotting Eq. (5) and Eq. (7). Figures 2 and 3 show an example of these 3D EXIT charts, when Encoder I is a half-rate memory-1 recursive systematic convolutional (RSC) code having octally represented generator polynomials of $\left(G_{r}, G\right)=(3,2)_{8}$, where $G_{r}$ is the feedback polynomial, while Encoder II is a simple rate- 1 accumulator, described by the pair of octal generator polynomials $\left(G_{r}, G\right)=(3,2)_{8}$.

\section{2D EXIT Chart Projections}

The 3D EXIT charts of Figures 2 and 3 are somewhat cumbersome to interpret as well as to plot. Hence in this section we derive their unique and unambiguous $2 \mathrm{D}$ representations, which can be interpreted in the usual way.

The intersection of the surfaces in Figure 2, shown as a thick solid line, portrays the best achievable performance, 


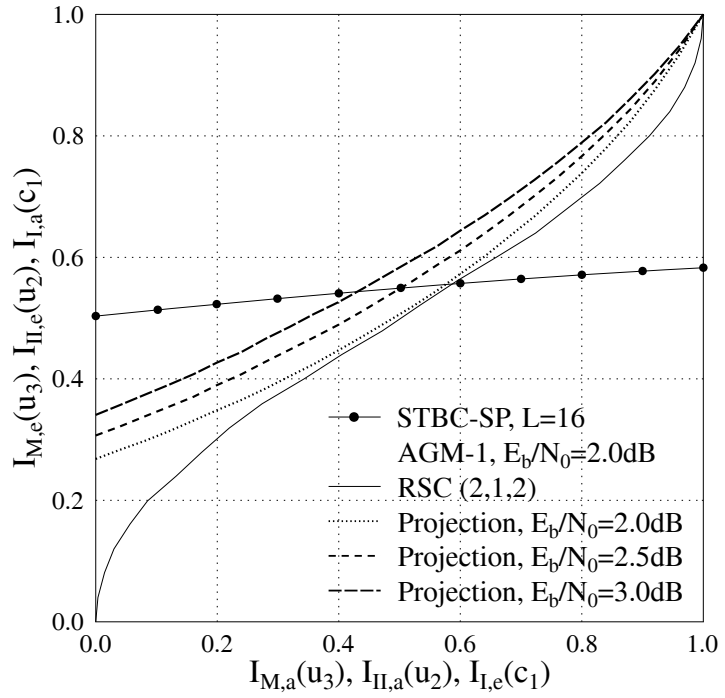

Fig. 4. 2D projection of the EXIT charts of the three-stage RSC-coded STBC-SP scheme, when employing the anti-Gray mapping AGM-1 of Table II.

when exchanging mutual information between the SP demapper and the rate-1 Decoder II for different fixed values of $I_{I I, a}\left(u_{2}\right)$ spanning the range of $[0,1]$. Each $\left(I_{I I, a}\left(u_{2}\right)\right.$, $\left.I_{I I, a}\left(c_{2}\right), I_{I I, e}\left(c_{2}\right)\right)$ point belonging to the intersection line in Figure 2 uniquely specifies a 3D point $\left(I_{I I, a}\left(u_{2}\right)\right.$, $\left.I_{I I, a}\left(c_{2}\right), I_{I I, e}\left(u_{2}\right)\right)$ in Figure 3 , according to the EXIT function of Eq. (5). Therefore, the line corresponding to the $\left(I_{I I, a}\left(u_{2}\right), I_{I I, a}\left(c_{2}\right), I_{I I, e}\left(c_{2}\right)\right)$ points along the thick line of Figure 2 is projected to the solid line shown in Figure 3, while the $2 \mathrm{D}$ projection of the solid line in Figure 3 at $I_{I I, a}\left(c_{2}\right)=0$ onto the plane spanned by the lines $\left(I_{I I, a}\left(u_{2}\right), I_{I I, e}\left(u_{2}\right)\right)$ and $\left(I_{I, e}\left(c_{1}\right), I_{I, a}\left(c_{1}\right)\right)$ is shown in Figure 4, represented by the dotted line at $E_{b} / N_{0}=2.0 \mathrm{~dB}$. This projected EXIT curve may be written as

$$
I_{I I, e}\left(u_{2}\right)=T_{I I, u_{2}}^{p}\left(I_{I I, a}\left(u_{2}\right), E_{b} / N_{0}\right) .
$$

Projected 2D EXIT charts of similar nature will be used throughout the rest of the paper for the sake of describing the convergence behaviour of the three-stage turbo-detected STBC-SP scheme. More details on the related 3D-to-2D EXIT chart projection are provided in [26].

Figure 4 shows the 2D-projected EXIT curve of the SP demapper, when operating at $E_{b} / N_{0}=2.0 \mathrm{~dB}$ and employing Anti-Gray Mapping ${ }^{2}$ (AGM-1) scheme, which is described in Appendix A and in Table II. The figure also shows the 2D-projected EXIT curve of the outer RSC Decoder I and the 2D-projected EXIT curves of the combined SP demapper and the rate-1 Decoder II at different $E_{b} / N_{0}$ values, when employing AGM-1 of Table II. Observe in Figure 4 that an open convergence tunnel is taking shape for the threestage scheme upon increasing the Signal-to-Noise Ratio (SNR) beyond $E_{b} / N_{0}=2.0 \mathrm{~dB}$. This implies that according to the predictions of the 2D EXIT chart seen in Figure 4, the iterative decoding process is expected to converge to the $(1.0,1.0)$

\footnotetext{
${ }^{2}$ Anti-Gray Mapping (AGM) is used here to refer to any non-Gray mapping scheme, while the specific scheme AGM-1 and other AGM schemes employed in this paper are described in Appendix A and Table II.
}

point and hence an infinitesimally low BER may be attained beyond $E_{b} / N_{0}=2.0 \mathrm{~dB}$. By contrast, for the traditional two-stage turbo-detected STBC-SP scheme, there would be a BER floor preventing it from achieving an infinitesimally low BER due to the non-recursive nature of the SP demapper, which also prevents the intersection of the EXIT curves of the SP demapper and the outer RSC Decoder I from reaching the $(1.0,1.0)$ point of convergence, despite increasing the SNR or the number of iterations. In contrast to this, the three-stage scheme of Figure 1 becomes capable of achieving an infinitesimally low BER, as suggested by the EXIT-chart predictions of Figure 4.

\section{EXIT Tunnel-Area Minimisation for Near-Capacity Oper- ation}

In this section we will exploit the well-understood properties of conventional 2D EXIT charts that a narrow and open EXIT-tunnel represents a near-capacity performance. Therefore, we invoke Irregular Convolutional Codes (IRCCs) for the sake of appropriately shaping the EXIT curves by minimising the area within the EXIT-tunnel using the procedure of $[23$, 27].

Let $\mathcal{A}_{I}$ and $\overline{\mathcal{A}}_{I}$ be the areas under the EXIT-curve $T_{I, c_{1}}(i)$ of Eq. (7) and its inverse $T_{I, c_{1}}^{-1}(i), i \in[0,1]$, respectively, which are expressed as:

$$
\mathcal{A}_{I}=\int_{0}^{1} T_{I, c_{1}}(i) \mathrm{d} i, \quad \overline{\mathcal{A}}_{I}=\int_{0}^{1} T_{I, c_{1}}^{-1}(i) \mathrm{d} i=1-\mathcal{A}_{I} .
$$

Similarly, the area $\mathcal{A}_{I I}^{p}$ is defined under the EXIT-curve $T_{I I, u_{2}}^{p}(i)$ of Eq. (8). It was observed in $[23,32]$ that for the APP-based outer Decoder I, the area $\overline{\mathcal{A}}_{I}$ maybe approximated by $\overline{\mathcal{A}}_{I} \approx R_{I}$, where the equality $\overline{\mathcal{A}}_{I}=R_{I}$ was later shown in [28] for the family of Binary Erasure Channels (BECs). The area property of $\overline{\mathcal{A}}_{I} \approx R_{I}$ implies that the lowest SNR convergence threshold occurs, when we have $\mathcal{A}_{I I}^{p}=R_{I}+\epsilon$, where $\epsilon$ is an infinitesimally small number, provided that the following convergence constraints hold [27]:

$$
\begin{aligned}
& T_{I I, u_{2}}^{p}(0)>0, \quad T_{I I, u_{2}}^{p}(1)=1, \quad \text { and } \\
& T_{I I, u_{2}}^{p}(i)>T_{I, c_{1}}^{-1}(i), \quad \forall i \in[0,1) .
\end{aligned}
$$

Observe, in Figure 4, however that there is a 'larger-thannecessary' tunnel area between the projected EXIT curve $T_{I I, u_{2}}^{p}(i)$ and the EXIT curve $T_{I, c_{1}}^{-1}(i)$ of the outer $1 / 2$-rate RSC code at $E_{b} / N_{0}=2.0 \mathrm{~dB}$. This implies that the BER curve is farther from the achievable capacity than necessary, despite the fact that the specific bit-to-SP-symbol mapping scheme of AGM-1 and the 1/2-rate RSC code employed in Figure 4 were specifically optimised for convergence at a low $E_{b} / N_{0}$ value. More quantitatively, the area under the projected EXIT curve $T_{I I, u_{2}}^{p}(i)$ is $\mathcal{A}_{I I}^{p} \approx 0.55$ at $E_{b} / N_{0}=2.0 \mathrm{~dB}$, which is larger than the outer code rate of $R_{I}=0.50$. Therefore, according to Figure 4 and to the area property of $\overline{\mathcal{A}}_{I} \approx R_{I}$, a lower $E_{b} / N_{0}$ convergence threshold may be attained, provided that the constraints outlined in Eq. (10) are satisfied. Hence we will invoke IRCCs [23,27] as outer codes that exhibit flexible EXIT characteristics, which can be optimised to more closely match the 2D-projected EXIT 


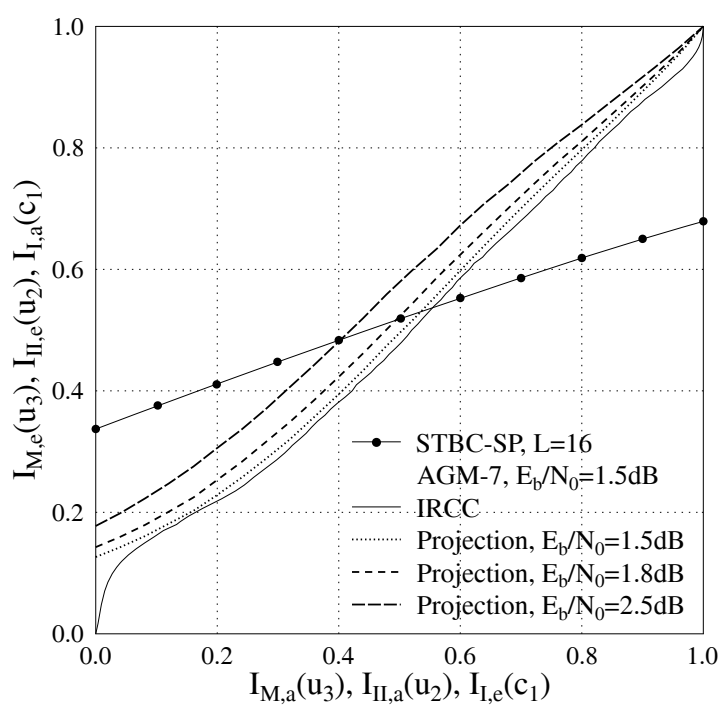

Fig. 5. 2D projection of the EXIT charts of the three-stage IRCCcoded STBC-SP scheme, when employing the anti-Gray mapping AGM-2 of Table II.

curve $T_{I I, u_{2}}^{p}(i)$ of Figure 4, rendering the near-capacity code optimisation a simple curve-fitting process.

An IRCC scheme constituted by a set of $P=17$ subcodes was constructed in [27] from a systematic $1 / 2$-rate memory4 mother code defined by the octally represented generator polynomials $\left(G_{r}, G\right)=(31,27)_{8}$. Each of the $P=17$ subcodes encodes a specific fraction of the uncoded bits determined by the weighting coefficient, $\alpha_{i}, i=1, \ldots, P$. Hence the coefficients $\alpha_{i}$ are optimised with the aid of the iterative algorithm of [23], so that the EXIT curve of the resultant IRCC closely matches the 2D-projected EXIT curve $T_{I I, u_{2}}^{p}(i)$ at the specific $E_{b} / N_{0}$ value, where we have $\mathcal{A}_{I I}^{p} \approx 0.50$. Observe in Figure 5 that we have $\mathcal{A}_{I I}^{p} \approx 0.51$ at $E_{b} / N_{0}=1.5 \mathrm{~dB}$, indicating that this $E_{b} / N_{0}$ value is close to the lowest attainable convergence threshold, when employing a $1 / 2$-rate outer code. Figure 5 also shows the 2D-projected EXIT curve of the resultant IRCC, where the optimised weighting coefficients are as follows:

$$
\begin{aligned}
{\left[\alpha_{1}, \ldots, \alpha_{17}\right]=} & {[0,0.0559066,0.236757,} \\
& 0,0,0,0.23844,0,0, \\
& 0.0306247,0,0.205574,0, \\
& 0,0.110076,0,0.122621] .
\end{aligned}
$$

\section{Capacity and Maximum Achievable Rate}

\section{A. Capacity of STBC-SP Schemes}

For the sake of simplicity, a system having two transmit and a single receive antenna is considered, although its extension to systems having more than one receive antenna is straightforward. Assuming perfect channel estimation, the complex-valued channel output symbols received during two consecutive STBC time slots are first diversity-combined in order to extract the estimates $\tilde{x}_{1}$ and $\tilde{x}_{2}$ of the most likely transmitted symbols $x_{l, 1}$ and $x_{l, 2}$ [4][1, pp. 400-401], resulting in

$$
\begin{aligned}
& \tilde{x}_{1}=\left(\left|h_{1}\right|^{2}+\left|h_{2}\right|^{2}\right) \cdot x_{l, 1}+\dot{n}_{1} \\
& \tilde{x}_{2}=\left(\left|h_{1}\right|^{2}+\left|h_{2}\right|^{2}\right) \cdot x_{l, 2}+\dot{n}_{2},
\end{aligned}
$$

where $h_{1}$ and $h_{2}$ represent the complex-valued non-dispersive channel coefficients corresponding to the first and second transmit antenna, respectively, and $\dot{n}_{1}$ as well as $\dot{n}_{2}$ are zero-mean complex Gaussian random variables with variance $\sigma_{\tilde{n}}^{2}=\left(\left|h_{1}\right|^{2}+\left|h_{2}\right|^{2}\right) \cdot \sigma_{n}^{2}$. A received sphere-packed symbol $\mathbf{r}$ is then constructed from the estimates $\tilde{x}_{1}$ and $\tilde{x}_{2}$ using Eq. (2) as

$$
\mathbf{r}=T_{s p}^{-1}\left(\tilde{x}_{1}, \tilde{x}_{2}\right),
$$

where $\mathbf{r}=\left[\begin{array}{llll}\tilde{a_{1}} & \tilde{a_{2}} & \tilde{a_{3}} & \tilde{a_{4}}\end{array}\right] \in \mathbb{R}^{4}$. The received sphere-packed symbol $\mathbf{r}$ can be written as

$$
\mathbf{r}=h \cdot \sqrt{\frac{2 L}{E}} \cdot \mathbf{s}^{l}+\mathbf{w},
$$

where $h=\left(\left|h_{1}\right|^{2}+\left|h_{2}\right|^{2}\right), \mathbf{s}^{l} \in S, 0 \leq l \leq L-1$, and $\mathbf{w}$ is a four-dimensional real-valued Gaussian random variable having a covariance matrix of $\sigma_{w}^{2} \cdot \mathbf{I}_{N_{D}}=\sigma_{\tilde{n}}^{2} \cdot \mathbf{I}_{N_{D}}=h \cdot \sigma_{n}^{2} \cdot \mathbf{I}_{N_{D}}$, where we have $N_{D}=4$, since the symbol constellation $S$ is four-dimensional. According to Eq. (15), the conditional PDF $p\left(\mathbf{r} \mid \mathbf{s}^{l}\right)$ is given by

$$
\begin{aligned}
p\left(\mathbf{r} \mid \mathbf{s}^{l}\right) & =\frac{1}{\left(2 \pi \sigma_{w}^{2}\right)^{\frac{N_{D}}{2}}} e^{-\frac{1}{2 \sigma_{w}^{2}}\left(\mathbf{r}-\alpha \cdot \mathbf{s}^{l}\right)\left(\mathbf{r}-\alpha \cdot \mathbf{s}^{l}\right)^{T}}, \\
& =\frac{1}{\left(2 \pi \sigma_{w}^{2}\right)^{\frac{N_{D}}{2}}} e^{-\frac{1}{2 \sigma_{w}^{2}}\left(\sum_{i=1}^{4}\left(\tilde{a}_{i}-\alpha \cdot a_{l, i}\right)^{2}\right)},
\end{aligned}
$$

where we have $\alpha=h \cdot \sqrt{\frac{2 L}{E}}$ and $(\cdot)^{T}$ represents the transpose of a vector.

The channel capacity valid for STBC schemes using $N_{D}$-dimensional so-called $L$-orthogonal signalling [33] over the Discrete-input Continuous-output Memoryless Channel (DCMC) [34] was derived in [35]. Assuming that all the legitimate transmitted 4-dimensional SP symbols, $\mathbf{s}^{l} \in S$, $0 \leq l \leq L-1$, are equiprobable, the channel capacity of the STBC-SP scheme over the DCMC is given by $[35,36]$

$$
C_{D C M C}^{S T B C-S P}=\log _{2}(L)-\frac{1}{L} \sum_{l=0}^{L-1} E\left[\log _{2} \sum_{k=0}^{L-1} e^{\Psi_{l, k}} \mid \mathbf{s}^{l}\right],
$$

where $E\left[\xi \mid \mathbf{s}^{l}\right]$ is the expectation of $\xi$ conditioned on $\mathbf{s}^{l}$ and

$$
\Psi_{l, k}=\sum_{d=1}^{N_{D}}\left(\frac{\left(\alpha \cdot\left(\mathbf{s}^{l}[d]-\mathbf{s}^{k}[d]\right)+\mathbf{w}[d]\right)^{2}+(\mathbf{w}[d])^{2}}{2 \sigma_{w}^{2}}\right),
$$

with $N_{D}=4$. The bandwith-efficiency was defined as [35]

$$
\eta_{D C M C}^{S T B C-S P}\left(E_{b} / N_{0}\right)=\frac{C_{D C M C}^{S T B C-S P}}{N_{D} / 2}=\frac{C_{D C M C}^{S T B C-S P}}{2} .
$$

Figure 6 shows the DCMC capacity of the 4-dimensional SP modulation assisted STBC scheme for $L=16$, where the Continuous-Input Continuous-Output Memoryless Channel (CCMC) [34] capacity of the MIMO scheme is given by [37]. More specifically, Figure 6 demonstrate that at a 


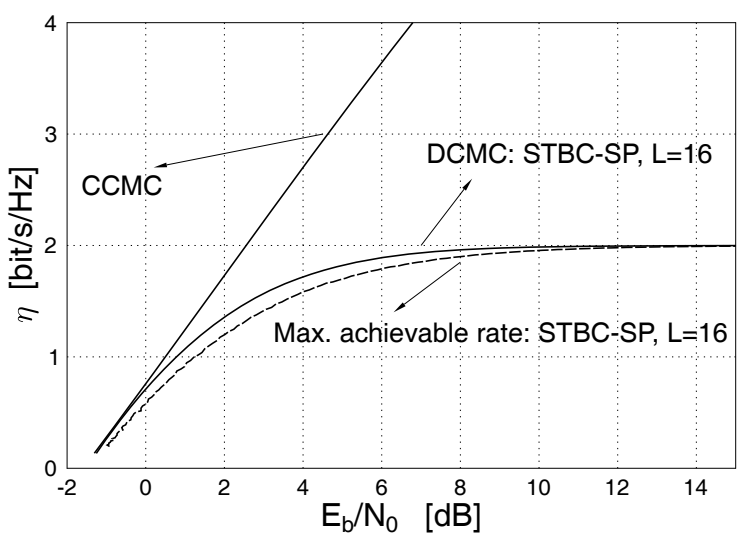

Fig. 6. Bandwidth efficiency of the STBC-SP based system with $L=16$, when employing $N_{t}=2$ transmit antennas and $N_{r}=1$ receive antenna.

bandwidth efficiency of $\eta=1 \mathrm{bit} / \mathrm{s} / \mathrm{Hz}$, the capacity limit for the DCMC STBC-SP scheme employing $N_{t}=2$ transmit and $N_{r}=1$ receive antennas is $E_{b} / N_{0}=0.78 \mathrm{~dB}$. The EXIT chart analysis of Figure 5 predicts that our three-stage system will converge at $E_{b} / N_{0}=1.5 \mathrm{~dB}$, i.e. within $0.72 \mathrm{~dB}$ from the capacity limit. The dashed curve in Figure 6 that refers to the maximum achievable rate of the three-stage turbo-detected STBC-SP scheme is discussed next in Section IV-B.

\section{B. Maximum Achievable Rate}

A tighter upper limit on the maximum achievable rate of the system can be calculated based on the area property of $\overline{\mathcal{A}}_{I} \approx R_{I}$ the EXIT charts discussed in Section III-III-D. More explicitly, it was shown in Section III-III-D that the outer Decoder I may have a maximum rate of $R_{I}^{\max } \approx \mathcal{A}_{I I}^{p}$ at a specific $E_{b} / N_{0}$ value, where $\mathcal{A}_{I I}^{p}$ is the area under the projected EXIT curve of the SP demapper and the rate-1 Decoder II of Eq. (8). Therefore, if $\mathcal{A}_{I I}^{p}$ is calculated for different $E_{b} / N_{0}$ values, the maximum achievable bandwidth efficiency may be formulated as a function of the $E_{b} / N_{0}$ value as follows

$$
\begin{aligned}
\eta_{\max }\left(\underline{\left.E_{b} / N_{0}\right)}\right. & =B \cdot R_{S T B C-S P} \cdot R_{I}^{\max } \\
& \approx B \cdot R_{S T B C-S P} \cdot \mathcal{A}_{I I}^{p}\left(E_{b} / N_{0}\right),
\end{aligned}
$$

where $B=\log _{2}(L)$ is the number of bits per SP symbol and $R_{S T B C-S P}=\frac{1}{2}$, since $T=2$ time slots are needed to transmit one SP symbol according to Eqs. (1) and (3). Additionally, $E_{b} / N_{0}$ and $E_{b} / N_{0}$ are related as follows

$$
\underline{E_{b} / N_{0}}=E_{b} / N_{0}+10 \log \left(\frac{R_{o}}{\mathcal{A}_{I I}^{p}\left(E_{b} / N_{0}\right)}\right) \quad[\mathrm{dB}],
$$

where $R_{O}$ is the original outer code rate used when generating the 2D-projected EXIT curves of the SP demapper and the rate-1 Decoder II of Eq. (8) corresponding to the different $\mathcal{A}_{I I}^{p}$ values. Basically, $E_{b} / N_{0}$ refers to the effective $E_{b} / N_{0}$ corresponding to the maximum accommodated outer Decoder I rate $R_{I}$. A simple procedure may be used to calculate the maximum achievable bandwith efficiency of Eq. (20). For computational simplicity, the area $\mathcal{A}_{M}$ under the EXIT curve $T_{M, u_{3}}$ of Eq. (6) may be used instead of the area $\mathcal{A}_{I I}^{p}$ under the
2D-projected EXIT curve $T_{I I, u_{2}}^{p}$ of Eq. (8), since $\mathcal{A}_{M}=\mathcal{A}_{I I}^{p}$, when $R_{I I}=1$. More specifically, the maximum achievable bandwith efficiency of Eq. (20) can be calculated using the following procedure for $E_{b} / N_{0} \in\left[\rho_{\min }, \rho_{\max }\right]$, assuming that $R_{o}$ is an arbitrary rate and $\epsilon$ is a small constant.

Algorithm 1 (Maximum Achievable Bandwidth Efficiency using EXIT Charts):

Step 1: Let $R_{I}=R_{o}$.

Step 2: Let $E_{b} / N_{0}=\rho_{\min } \mathrm{dB}$.

Step 3: Calculate $N_{0}$.

Step 4: Let $I_{M, a}\left(u_{3}\right)=0$.

Step 5: Activate the SP demapper.

Step 6: Save $I_{M, e}\left(u_{3}\right)=T_{M, u_{3}}\left(I_{M, a}\left(u_{3}\right), E_{b} / N_{0}\right)$.

Step 7: Let $I_{M, a}\left(u_{3}\right)=I_{M, a}\left(u_{3}\right)+\epsilon$. If $I_{M, a}\left(u_{3}\right) \leq 1.0$ go to Step 5.

Step 8: Calculate $\mathcal{A}_{M}\left(E_{b} / N_{0}\right)=\int_{0}^{1} T_{M, u_{3}}\left(i, E_{b} / N_{0}\right) \mathrm{d} i$.

Step 9: Calculate $E_{b} / N_{0}$ using Eq. (21).

Step 10: Save $\eta_{\max } \overline{\left(E_{b} / N_{0}\right)}$ of Eq. (20).

Step 11: Let $E_{b} / N_{0} \overline{\overline{=E_{b} /}} N_{0}+\epsilon$. If $E_{b} / N_{0} \leq \rho_{\max } \mathrm{dB}$, go to Step 3.

Step 12: Output $\eta_{\max }\left(\underline{E_{b} / N_{0}}\right)$ from Step 10.

Observe that $\rho_{\min }$ and $\rho_{\max }$ are adjusted accordingly in order to produce the desired range of the resultant $E_{b} / N_{0}$ values. Furthermore, the output of Algorithm 1 is independent of the specific choice of $R_{o}$, since Eq. (21) would always adjust the $E_{b} / N_{0}$ values, regardless of $R_{o}$. For example, $R_{o}$ may be set to the desired final $R_{I}$ to be employed in the three-stage system.

The resultant maximum achievable bandwidth efficiency is demonstrated in Figure 6, which is slightly lower than the bandwidth efficiency of Eq. (19), i.e. we have $\eta_{\max }<$ $\eta_{D C M C}^{S T B C-S P}$. Observe that the bandwidth efficiency calculated using Eq. (19) and using the EXIT charts as well as Eq. (20) were only proven to be equal for the family of BECs [28]. Nonetheless, similar trends have been observed for both AWGN and Inter-Symbol-Interference (ISI) channels [25, 27], when APP-based decoders are used for all decoder blocks [28]. However, the discrepancy between the two bandwidth efficiency curves shown in Figure 6 that are calculated using Eq. (19) and Eq. (20) is due to the fact that the SP demapper is not an APP-based decoder. Nevertheless, the bandwidth efficiency calculated based on the EXIT charts using Eq. (20) and Algorithm 1 constitutes a tighter bound on the maximum achievable bandwidth efficiency of the system.

Figure 6 shows that at a bandwidth efficiency of $\eta=1$ $\mathrm{bit} / \mathrm{s} / \mathrm{Hz}$, the capacity limit for the STBC-SP scheme is about $E_{b} / N_{0}=1.3 \mathrm{~dB}$, which is within $0.2 \mathrm{~dB}$ from the prediction of our EXIT chart analysis seen in Figure 5, where convergence is predicted at $E_{b} / N_{0}=1.5 \mathrm{~dB}$.

\section{RESUlts AND Discussions}

Without loss of generality, we considered a sphere packing modulation scheme associated with $L=16$ using two transmit and a single receiver antenna in order to demonstrate the performance improvements achieved by the proposed system. All simulation parameters are listed in Table I.

\section{A. Decoding Trajectory}

EXIT chart based convergence predictions are usually verified by the actual iterative decoding trajectory. Figure 5 shows 
TABLE I

SYSTEM PARAMETERS.

\begin{tabular}{|l|r|}
\hline Modulation & Sphere Packing with $L=16$ \\
\hline No. of transmitters & 2 \\
\hline No. of receivers & Time-correlated Rayleigh Fading \\
\hline Channel & 0.1 \\
\hline $\begin{array}{l}\text { Normalised Doppler } \\
\text { frequency }\end{array}$ & $\begin{array}{r}\text { half-rate memory-4 IRCC, } P=17, \\
\text { weighting coefficients of Eq. }(11)\end{array}$ \\
\hline Outer Encoder I & $\begin{array}{r}\text { Rate-1 memory-1 RSC code, } \\
\left(G_{r}, G\right)=(3,2)_{8}\end{array}$ \\
\hline Rate-1 Encoder II & $\begin{array}{r}\text { SP demapper } \Rightarrow \text { Decoder II } \Rightarrow \\
\text { SP demapper } \Rightarrow \text { Decoder II } \Rightarrow \\
\text { Decoder I } \Rightarrow \text { Decoder II }\end{array}$ \\
\hline $\begin{array}{l}\text { Activation sequence of } \\
\text { a three-stage iteration }\end{array}$ & 1 bit/symbol \\
\hline System throughput &
\end{tabular}

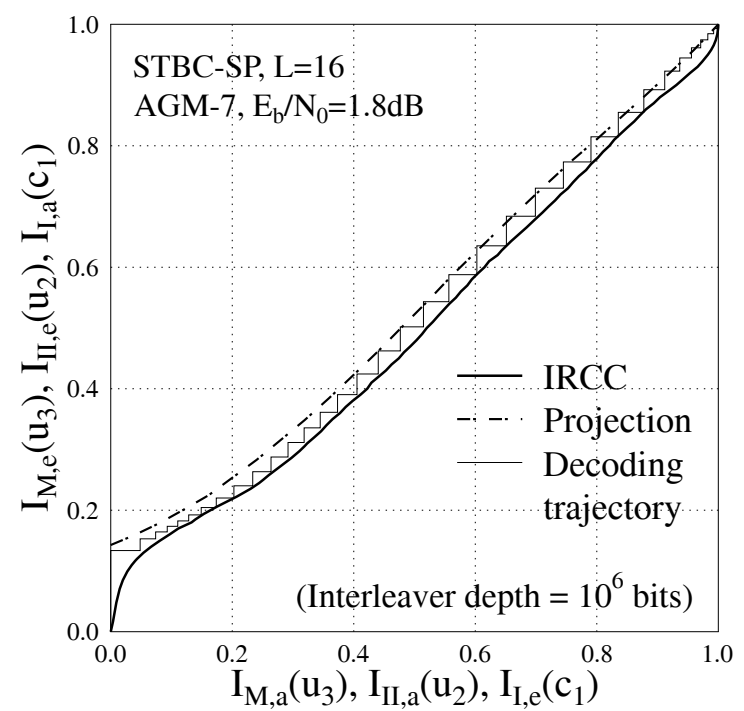

Fig. 7. Decoding trajectory of the three-stage IRCC-coded STBC-SP scheme, when employing the anti-Gray mapping AGM-2 of Table II in combination with the system parameters outlined in Table I and operating at $E_{b} / N_{0}=1.8 \mathrm{~dB}$ with an interleaver depth of $\mathcal{D}=10^{6}$ bits after 33 three-stage iterations.

that the three-stage turbo-detected STBC-SP scheme is expected to converge at $E_{b} / N_{0}=1.5 \mathrm{~dB}$, where convergence to the $(1.0,1.0)$ point requires an excessive number of three-stage iterations. However, convergence to the $(1.0,1.0)$ point becomes more feasible for $E_{b} / N_{0}>1.5 \mathrm{~dB}$. Figure 7 illustrates the actual decoding trajectory of the three-stage turbo-detected STBC-SP scheme of Figure 1 at $E_{b} / N_{0}=1.8 \mathrm{~dB}$, when using an interleaver depth of $\mathcal{D}=10^{6}$ bits and 33 three-stage iterations. The zigzag-path seen in Figure 7 represents the actual extrinsic information transfer between the SP demapper and the rate-1 Decoder II on one hand and the outer IRCC Decoder I on the other.

\section{B. BER Performance}

Figure 8 compares the performance of the proposed threestage IRCC-coded STBC-SP scheme employing anti-Gray mapping (AGM-2) against that of an identical-throughput 1 Bit Per Symbol (1BPS) uncoded STBC-SP scheme [3] using $L=4$ and against Alamouti's conventional $\mathbf{G}_{2}$-BPSK scheme [4]. The system is also benchmarked against a twostage RSC-coded STBC-SP scheme [13], when employing the

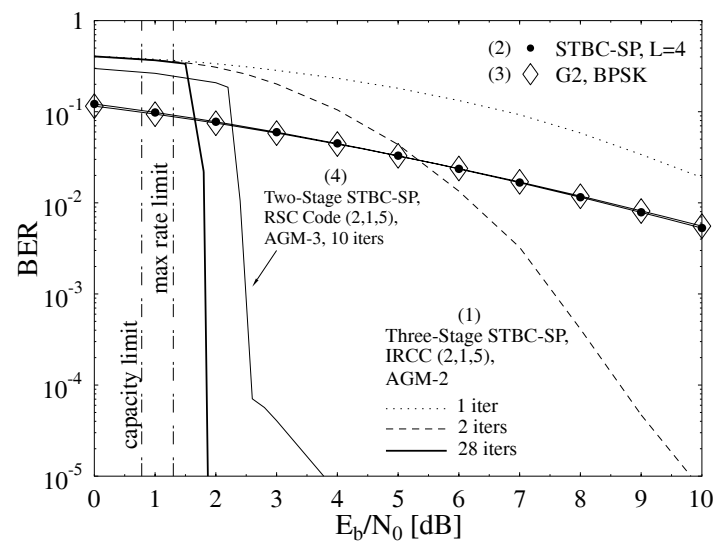

Fig. 8. Performance comparison of the anti-Gray mapping AGM-2 ${ }^{(1)}$ based IRCC-coded three-stage STBC-SP scheme in conjunction with $L=$ 16 against an identical-throughput 1 bit/symbol (BPS) uncoded STBC-SP scheme $^{(2)}$ using $L=4$ and against Alamouti's conventional $\mathbf{G}_{2}$-BPSK scheme $^{(3)}$ as well as against a two-stage RSC-coded STBC-SP scheme ${ }^{(4)}$, when employing the system parameters outlined in Table I and using an interleaver depth of $\mathcal{D}=10^{6}$ bits.

system parameters outlined in Table I and using an interleaver depth of $\mathcal{D}=10^{6}$ bits. Figure 8 specifically demonstrates that the proposed three-stage turbo-detected scheme is capable of achieving infinitesimally low BER values, where its performance is not limited by a BER floor, which is in contrast to the two-stage turbo-detected STBC-SP scheme. Observe that the two-stage turbo-detected STBC-SP scheme uses only 10 iterations since the advantage of employing any further iterations diminishes owing to the presence of a BER floor. Explicitly, Figure 8 demonstrates that a coding advantage of about $22.2 \mathrm{~dB}$ was achieved at a BER of $10^{-5}$ after 28 iterations by the three-stage turbo-detected STBC-SP system over both the uncoded STBC-SP [3] and the conventional orthogonal STBC design based $[4,5]$ schemes for transmission over the correlated Rayleigh fading channel considered. Additionally, a coding advantage of approximately $2.0 \mathrm{~dB}$ was attained over the 1BPS-throughput RSC-coded AGM-3 STBC-SP scheme [13] at the expense of an increased decoding complexity due to the employment of the rate- 1 decoder and the additional three-stage iterations. According to Figure 8, the three-stage turbo-detected STBC-SP scheme operates within approximately $1.0 \mathrm{~dB}$ from the capacity limit of Eq. (19) and $0.5 \mathrm{~dB}$ from the maximum achievable bandwidth efficiency limit of Eq. (20).

\section{Effect of Interleaver Depth}

The EXIT chart predictions are typically closely met, when large interleaver depths are employed, as mentioned in Section III-III-A. Moreover, for a practical perspective it is always interesting to investigate the achievable performance, when employing shorter interleaver depths, while using different number of three-stage iterations. Figure 9 shows the achievable coding gain of the three-stage STBC-SP scheme against Alamouti's conventional identical-throughput 1 BPS $\mathbf{G}_{2}$-BPSK scheme, when employing various interleaver depths and different number of three-stage iterations. 


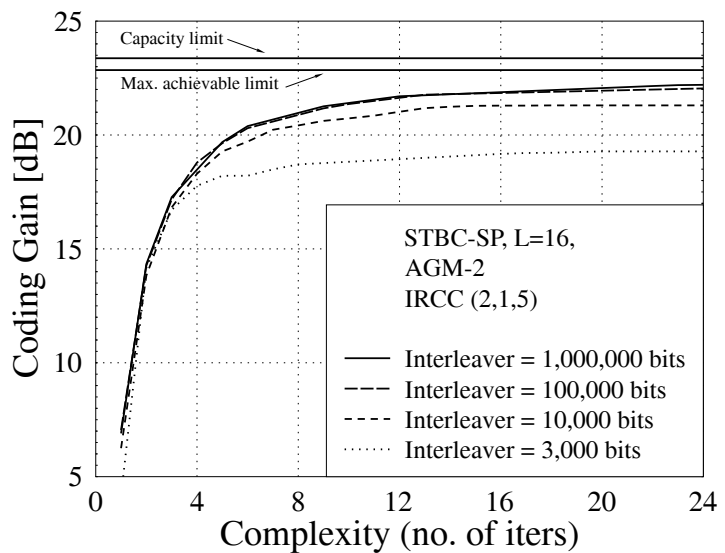

Fig. 9. Achievable coding gain of the three-stage STBC-SP scheme against Alamouti's identical-throughput (1 BPS) conventional $\mathbf{G}_{2}$-BPSK scheme, when employing various interleaver depths and different number of threestage iterations.

\section{CONCLUSION}

We proposed a three-stage serial concatenated turbodetected STBC-SP scheme that is capable of achieving infinitesimally low BER values, where the performace is not limited by a BER floor, which is routinely encountered in conventional two-stage systems, especially when the inner stage is constituted by a non-recursive inner decoder. The convergence behaviour of the three-stage system was analysed with the aid of novel 3D EXIT charts and their 2D projections $[25,26]$. With the advent of $2 \mathrm{D}$ projections, an IRCC $[23,27]$ was constructed for the sake of matching the projected EXIT curve of the SP demapper and the rate- 1 inner decoder leading to a near-capacity performance. The capacity of the STBC-SP scheme was calculated and a procedure was proposed for calculating a tighter upper bound on the maximum achievable bandwidth efficiency of the three-stage system using EXIT chart analysis. Our proposed three-stage scheme operated within about $1.0 \mathrm{~dB}$ from the capacity limit and within $0.5 \mathrm{~dB}$ from the maximum achievable bandwidth efficiency limit.

\section{APPENDIX A:}

\section{Anti-Gray Mapping Schemes for Sphere Packing Modulation OF Size $L=16$}

In this appendix, the different anti-Gray mapping schemes introduced in this paper for STBC-SP signal sets of size $L=16$ are described in detail. Since the space-time signal, which is constructed from an orthogonal design using the sphere packing scheme of Eq. (1) is multiplied by a factor that is inversely proportional to $\sqrt{E}$, namely by $\sqrt{\frac{2 L}{E}}$, it is desirable to choose a specific subset of $L=16$ points from the entire set of legitimate constellation points hosted by $D_{4}$, which results in the minimum total energy. It was shown in [29] that there is a total of 24 legitimate symbols hosted by $D_{4}$ having an identical minimum energy of $E=2$. In simple terms, the sphere centred at $(0,0,0,0)$ has 24 spheres around it, centred at the points $(+/-1,+/-1,0,0)$, where any choice of signs and any ordering of the coordinates is legitimate $[26$, p. 9]. We used a computer search for determining the optimum choice of the $L=16$ points out of the possible
TABLE II

BIT MAPPINGS FOR THE DIFFERENT ANTI-GRAY MAPPING SCHEMES INTRODUCED FOR STBC-SP SIGNALS OF SIZE $L=16$.

\begin{tabular}{|c|c|c|c||c|c|c|}
\hline \multicolumn{4}{|c||}{ Points from $D_{4}$} & \multicolumn{3}{c|}{ Integer Index } \\
\hline$a_{1}$ & $a_{2}$ & $a_{3}$ & $a_{4}$ & AGM-1 & AGM-2 & AGM-3 \\
\hline+1 & -1 & 0 & 0 & 0 & 2 & 12 \\
\hline 0 & -1 & -1 & 0 & 1 & 4 & 11 \\
\hline 0 & -1 & +1 & 0 & 2 & 5 & 7 \\
\hline-1 & -1 & 0 & 0 & 3 & 0 & 15 \\
\hline-1 & 0 & 0 & +1 & 4 & 13 & 14 \\
\hline 0 & 0 & -1 & +1 & 5 & 9 & 5 \\
\hline 0 & 0 & +1 & +1 & 6 & 11 & 9 \\
\hline+1 & 0 & 0 & +1 & 7 & 15 & 2 \\
\hline-1 & 0 & 0 & -1 & 8 & 12 & 13 \\
\hline 0 & 0 & -1 & -1 & 9 & 8 & 6 \\
\hline 0 & 0 & +1 & -1 & 10 & 10 & 10 \\
\hline+1 & 0 & 0 & -1 & 11 & 14 & 1 \\
\hline-1 & +1 & 0 & 0 & 12 & 1 & 3 \\
\hline 0 & +1 & -1 & 0 & 13 & 6 & 8 \\
\hline 0 & +1 & +1 & 0 & 14 & 7 & 4 \\
\hline+1 & +1 & 0 & 0 & 15 & 3 & 0 \\
\hline
\end{tabular}

24 points, which possess the highest minimum Euclidean distance, hence minimising the error probability. All mapping schemes described here use the same 16 optimum constellation points. More specifically, for all mapping schemes, constellation points of the lattice $D_{4}$ are given for each integer index $l=0,1, \ldots, 15$. The normalisation factor of these constellation points is $\sqrt{\frac{2 L}{E}}=1$. The constellation points corresponding to each mapping scheme are given in Table II.

\section{REFERENCES}

[1] L. Hanzo, T. H. Liew, and B. L. Yeap, Turbo Coding, Turbo Equalisation and Space-Time Coding for Transmission over Fading Channels. Chichester, England: John Wiley and Sons Ltd and IEEE Press, 2002.

[2] L. Hanzo, O. R. Alamri, M. El-Hajjar, and N. Wu, Near-Capacity Multi-Functional MIMO Systems: Sphere-Packing, Iterative Detection and Cooperation. Wiley, 2009.

[3] W. Su, Z. Safar, and K. J. R. Liu, "Space-time signal design for time-correlated Rayleigh fading channels," in Proc. IEEE International Conference on Communications, vol. 5, Anchorage, Alaska, 2003, pp. 3175-3179.

[4] S. Alamouti, "A simple transmit diversity technique for wireless communications," IEEE J. Select. Areas Commun., vol. 16, no. 8, pp. 14511458, 1998.

[5] V. Tarokh, H. Jafarkhani, and A. Calderbank, "Space-time block codes from orthogonal designs," IEEE Trans. Inform. Theory, vol. 45, no. 5, pp. 1456-1467, July 1999.

[6] C. Berrou, A. Glavieux, and P. Thitimajshima, "Near Shannon limit error-correcting coding and decoding: Turbo codes," in Proc. IEEE International Conference on Communications, pp. 1064-1070, May 1993.

[7] D. Divsalar and F. Pollara, "Multiple turbo codes for deep-space communications," Jet Propulsion Laboratory, Pasadena, CA, Telecommunications and Data Acquisition Progress Report 42-121, May 1995.

[8] S. Benedetto and G. Montorsi, "Serial concatenation of block and convolutional codes," IEE Electron. Lett., vol. 32, no. 10, pp. 887-888, 1996.

[9] S. Benedetto, D. Divsalar, G. Montorsi, and F. Pollara, "Analysis, design, and iterative decoding of double serially concatenated codes with interleavers," IEEE J. Select. Areas Commun., vol. 16, no. 2, pp. 231-244, 1998.

[10] M. Breiling and L. Hanzo, "The super-trellis structure of turbo codes," IEEE Trans. Inform. Theory, vol. 46, no. 6, pp. 2212-2228, Sept. 2000.

[11] S. ten Brink, J. Speidel, and R.-H. Yan, "Iterative demapping and decoding for multilevel modulation," in Proc. IEEE Global Telecommunications Conference, vol. 1, Sydney, Australia, 8-12 Nov. 1998, pp. $579-584$.

[12] A. Sezgin, D. Wuebben, and V. Kuehn, "Analysis of mapping strategies for turbo-coded space-time block codes," in Proc. IEEE Information Theory Workshop, Mar. 2003, pp. 103-106. 
[13] O. Alamri, B. L. Yeap, and L. Hanzo, "A turbo detection and sphere packing modulation aided space-time coding scheme," IEEE Trans. Veh. Technol., vol. 56, no. 2, pp. 575-582, Mar. 2007.

[14] O. Alamri, S.-X. Ng, F. Guo, S. Zummo, and L. Hanzo, "Nonbinary LDPC-coded sphere-packed transmit diversity," IEEE Trans. Veh. Technol., vol. 57, pp. 3200-3205, Sept. 2008.

[15] M. El-Hajjar, O. Alamri, S-X. Ng, and L. Hanzo, "Turbo detection of precoded sphere packing modulation using four transmit antennas for differential space-time spreading," IEEE Trans. Wireless Commun., vol. 7, pp. 943-952, Mar. 2008.

[16] S. Benedetto, D. Divsalar, G. Montorsi, and F. Pollara, "Serial concatenation of interleaved codes: performance analysis, design, and iterative decoding," IEEE Trans. Inform. Theory, vol. 44, no. 3, pp. 909-926, 1998.

[17] D. Divsalar, S. Dolinar, and F. Pollara, "Serial concatenated trellis coded modulation with rate-1 inner code," in Proc. IEEE Global Telecommunications Conference, vol. 2, San Francisco, USA, Nov. 2000, pp. 777-782.

[18] K. Narayanan, "Effect of precoding on the convergence of turbo equalization for partial response channels," IEEE J. Select. Areas Commun., vol. 19 , no. 4, pp. 686-698, Apr. 2001.

[19] I. Lee, "The effect of a precoder on serially concatenated coding systems with an ISI channel," IEEE Trans. Commun., vol. 49, no. 7, pp. 11681175 , July 2001

[20] H. Tullberg and P. Siegel, "Serial concatenated trellis coded modulation with inner rate-1 accumulate code," in Proc. IEEE Global Telecommunications Conference 2001 GLOBECOM'01, vol. 2, San Antonio, TX, 2001, pp. 936-940.

[21] L. Li, D. Divsalar, and S. Dolinar, "Iterative demodulation, demapping, and decoding of coded non-square QAM," in IEEE Trans. Commun., vol. 53, no. 1, Jan. 2005, pp. 16-19.

[22] S. ten Brink, "Designing iterative decoding schemes with the extrinsic information transfer chart," AEÜ International J. Electron. and Commun., vol. 54, no. 6, pp. 389-398, Nov 2000.

[23] M. Tüchler and J. Hagenauer, "EXIT charts of irregular codes," in Proc. 36th Annual Conference on Information Science and Systems, CISS 2002, Princeton, NJ, Mar. 2002.

[24] S. ten Brink, "Convergence of multi-dimensional iterative decoding schemes," in Proc. 35th Asilomar Conference on Signals, Systems, and Computers, vol. 1, Pacific Grove, CA, Nov. 2001, pp. 270-274.

[25] M. Tüchler, "Convergence prediction for iterative decoding of threefold concatenated systems," in Proc. IEEE Global Telecommunications Conference GLOBECOM'02, vol. 2, Nov. 2002, pp. 1358-1362.

[26] F. Brännström, L. Rasmussen, and A. Grant, "Convergence analysis and optimal scheduling for multiple concatenated codes," IEEE Trans. Inform. Theory, vol. 51, no. 9, pp. 3354-3364, 2005.

[27] M. Tüchler, "Design of serially concatenated systems depending on the block length," IEEE Trans. Commun., vol. 52, no. 2, pp. 209-218, Feb. 2004.

[28] A. Ashikhmin, G. Kramer, and S. ten Brink, "Extrinsic information transfer functions: model and erasure channel properties," IEEE Trans. Inform. Theory, vol. 50, no. 11, pp. 2657-2673, 2004.

[29] J. H. Conway and N. J. Sloane, Sphere Packings, Lattices and Groups. Springer-Verlag, 1999.

[30] W. C. Jakes, Microwave Mobile Communications. New York: Wiley, 1974.

[31] C. E. Shannon, "A mathematical theory of communication," Bell Systems Techn. J., vol. 27, pp. 379-423, 1948

[32] A. Ashikhmin, G. Kramer, and S. ten Brink, "Extrinsic information transfer functions: a model and two properties," in Proc. 36th Annual Conference on Information Sciences and Systems (CISS), Princeton, NJ, USA, Mar. 2002.

[33] L. Hanzo, S. X. Ng, T. Keller, and W. T. Webb, Quadrature Amplitude Modulation: From Basics to Adaptive Trellis-Coded, Turbo-Equalised and Space-Time Coded OFDM, CDMA and MC-CDMA Systems, 2nd ed. John Wiley and Sons Ltd., 2004.

[34] J. Proakis, Digital Communications. McGraw-Hill, 2001

[35] S. X. Ng and L. Hanzo, "On the MIMO channel capacity of multidimensional signal sets," IEEE Trans. Veh. Technol., vol. 55, no. 2, pp. 528-536, 2006

[36] E. Baccarelli, "Evaluation of the reliable data rates supported by multiple-antenna coded wireless links for QAM transmissions," IEEE J. Select. Areas Commun., vol. 19, no. 2, pp. 295-304, Feb. 2001.

[37] E. Telatar, "Capacity of multi-antenna Gaussian channels," European Trans. Telecommun., vol. 10, no. 6, pp. 585-595, Nov./Dec. 1999.

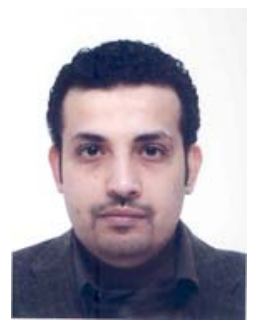

Osamah Rashed Alamri received his BS degree with first class honours in electrical engineering from King Fahd University of Petroleum and Minerals (KFUPM), Dhahran, Saudi Arabia, in 1997, where he was ranked first with a 4.0 GPA. Dr. Alamri received his MS degree in electrical engineering from Stanford University, California, USA, in 2002 and his Ph.D. degree in wireless communications from the University of Southampton, UK in 2007. He is currently a postdoctoral research fellow at the University of Southampton working on a collaborative European research project known as OPTIMIX. Dr. Alamri has published numerous papers and is a co-author of a forthcoming JohnWiley book on wireless communications. His research interests include sphere packing modulation, space-time coding, turbo coding and detection, multidimensional mapping and MIMO systems.

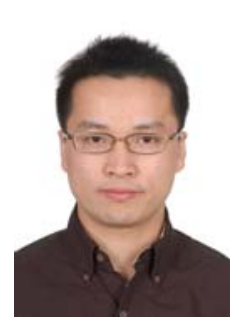

Jin Wang ( $\left.\mathrm{S}^{\prime} 03-\mathrm{M}^{\prime} 07\right)$ received the B.E. degree from University of Science and Technology of China (USTC) in 1999, the M.E. degree from Graduate School of Chinese Academy of Sciences (GSCAS) in 2002, and the PhD degree from University of Southampton, UK in 2007. In 2006, he joined Imagination Technologies Ltd as a software engineer, designing various video codecs. In 2008, he became an algorithm engineer for Aeroflex Cambridge Ltd. $\mathrm{He}$ is currently working on 3GPP HSPA and LTE receiver algorithms.

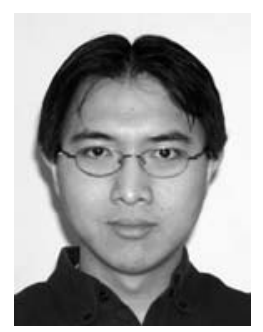

Soon Xin Ng (S'99-M'03) received the B.Eng. degree (First class) in electronics engineering and the Ph.D. degree in wireless communications from the University of Southampton, Southampton, U.K., in 1999 and 2002, respectively. From 2003 to 2006, he was a postdoctoral research fellow working on collaborative European research projects known as SCOUT, NEWCOM and PHOENIX. Since August 2006, he has been a lecturer in wireless communications at the University of Southampton. His research interests are mainly in adaptive coded modulation, channel coding, space-time coding, joint source and channel coding, OFDM and MIMO. He has published numerous papers and coauthored a book in this field.

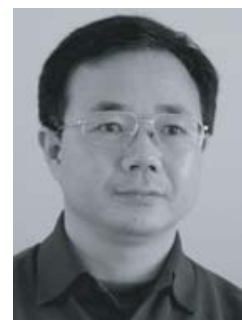

Lie-Liang Yang received his BEng degree in communications engineering from Shanghai TieDao University, Shanghai, China in 1988, and his MEng and $\mathrm{PhD}$ degrees in communications and electronics from Beijing (Northern) Jiaotong University, Beijing, China in 1991 and 1997, respectively. From June 1997 to December 1997 he was a visiting scientist of the Institute of Radio Engineering and Electronics, Academy of Sciences of the Czech Republic. Since December 1997, he has been with the School of Electronics and Computer Science, University of Southampton, UK, where he was first a Postdoctoral Research Fellow (Dec. 1997 - Aug. 2002), then a Lecturer (Sept. 2002 - Feb. 2006), and currently holds the academic post of Readership. Dr. Yang's research has covered a wide range of topics in wireless communications, networking and signal processing. He has published over 200 research papers in journals and conference proceedings, authored/co-authored two books and also published several book chapters. He was awarded the Royal Society Sino-British Fellowship in 1997 and the EPSRC Research Fellowship in 1998. Dr. Yang is currently an associate editor for the JOURNAL OF COMMUNICATIONS AND NeTWORKS (JCN) and the SECURITY AND COMMUNiCATION NETWORKS (SCN) JOURNAL. 


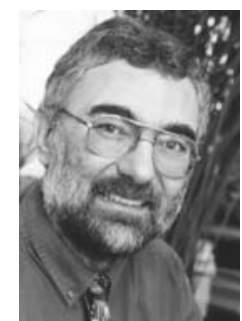

Lajos Hanzo , Fellow of the Royal Academy of Engineering, received his first-class degree in electronics in 1976 and his doctorate in 1983. In 2004 he was awarded the Doctor of Sciences (DSc) degree by the University of Southampton, UK. During his career in telecommunications he has held various research and academic posts in Hungary, Germany and the UK. Since 1986 he has been with the Department of Electronics and Computer Science, University of Southampton, UK, where he holds the chair in telecommunications. He has co-authored 17 books, totalling 10000 pages on mobile radio communications, published in excess of 800 research papers, has acted as TPC Chair of numerous major IEE and IEEE conferences, presented various keynote lectures and has been awarded a number of distinctions. Currently he heads an academic research team, working on a range of research projects in the field of wireless multimedia communications sponsored by industry, the Engineering and Physical Sciences Research Council (EPSRC) UK, the European IST Programme and the Mobile Virtual Centre of Excellence (VCE), UK. He is an enthusiastic supporter of industrial and academic liaison and he offers a range of industrial courses. Lajos is also an IEEE Distinguished Lecturer and a Governer of both the Communications as well as the Vehicular Technology Society, a Fellow of both the IEEE and the IEE. He is an editorial board member of the Proceedings of the IEEE and a Governor of both the IEEE Communications and VT Society. He was appointed as Editor-in-Chief of the IEEE Press for 2008 - 2009. For further information on research in progress and associated publications, please refer to http://www-mobile.ecs.soton.ac.uk. 